Pacific Journal of Mathematics

CHARACTERS OF AVERAGED DISCRETE SERIES ON 


\title{
CHARACTERS OF AVERAGED DISCRETE SERIES ON SEMISIMPLE REAL LIE GROUPS
}

\author{
REBECCA A. HeRB
}

\begin{abstract}
Let $G$ be a real simple Lie group of classical type having a compact Cartan subgroup. Then $G$ has discrete series representations. The purpose of this paper is to establish explicit formulas for certain sums of discrete series characters. These "averaged" discrete series characters have simple formulas which can be used for certain problems in harmonic analysis on $G$, for example, for the computation of the Plancherel measure on $\hat{G}$.
\end{abstract}

1. Introduction. Let $G$ be a connected, acceptable, semisimple real Lie group with finite center. Suppose that $G$ has a compact Cartan subgroup $T$. Then $G$ has discrete series representations. The characters of these representations were initially described by Harish-Chandra in [2]. The characters have simple formulas on $T$. On the noncompact Cartan subgroups, the formulas are complicated, and contain certain integer constants which Harish-Chandra did not compute.

Using the procedure described in [2], these constants can be computed if related constants are known for each type of simple root system which is spanned by a strongly orthogonal set of roots. These are the root systems of types $A_{1}, B_{n}, C_{n}, D_{2 n}(n \geqq 2), E_{7}, E_{8}, F_{4}$, and $G_{2}$, and they correspond to the complex simple Lie groups for which the split real form has a compact Cartan subgroup, and hence discrete series representations. Partial solutions to the problem of computing these constants have been given in $[4,5,6,7,8,10,11$, 12]. A complete solution is now available in work of $T$. Hirai [11]. Hirai's formulas express discrete series constants for groups of arbitrary rank in terms of constants for groups of real rank one and two.

Explicit formulas for discrete series characters, besides being of interest for the representation theory of $G$, are needed for harmonic analysis on $G$. However for some of these problems, for example, computation of the Plancherel measure on $\hat{G}$, it is necessary only to have certain sums of discrete series characters.

Let $g$ and $t$ denote the Lie algebras of $G$ and $T$ respectively, and $g_{c}$ and $t_{c}$ their complexifications. Then the discrete series characters of $G$ are parameterized by regular elements $\tau$ in a lattice $L_{T} \leqq$ $\sqrt{-1} t^{*}$. The Weyl group $W$ of the pair $\left(g_{c}, t_{c}\right)$ acts on $L_{T}$. Instead of the characters $(-1)^{q} \varepsilon(\tau) \theta_{\tau}$ defined by Harish-Chandra in [2], we 
consider the sum of characters

$$
(-1)^{q} \sum_{w \in W} \varepsilon(w \tau) \theta_{w \tau}=(-1)^{q} \varepsilon(\tau) \sum_{w \in W} \operatorname{det} w \theta_{w \tau} .
$$

Here $q=(1 / 2) \operatorname{dim}(G / K), K$ a maximal compact subgroup of $G$, and $\varepsilon(\tau)= \pm 1$ satisfies $\varepsilon(w \tau)=\operatorname{det} w \varepsilon(\tau)$. Note that if $\tau \in L_{T}$ is singular, invariant eigendistributions $\theta_{\tau}$ are defined by Harish-Chandra in [3]. However, for singular $\tau, \sum_{w \in W} \operatorname{det} w \theta_{w \tau}=0$.

These averaged discrete series characters are sufficient for the Fourier inversion of stabilized invariant integrals

$$
\mathscr{F}_{f}^{T}\left(t_{0}\right)=\sum_{w \in W} \operatorname{det} w F_{f}^{T}\left(w^{-1} t_{0}\right), f \in C_{o}^{\infty}(G), t_{0} \in T^{\prime} .
$$

Here $T^{\prime}$ is the set of regular elements in $T$ and $F_{f}^{T}$ is the invariant integral of $f$ with respect to $T$ defined in [1]. $F_{f}^{T}\left(t_{0}\right)$ can be regarded as the integral of $f$ over the orbit of $t_{0}$ in $G$ under the adjoint action of $G$. $\mathscr{F}_{f}^{T}\left(t_{0}\right)$ is the integral of $f$ over the orbit in $G$ under the adjoint action of $G_{c}$, a complex Lie group with Lie algebra $g_{c}$. Fourier inversion formulas for $\mathscr{F}_{f}^{T}\left(t_{0}\right)$ can be used to derive the Plancherel formula for $G$.

For the Fourier inversion of $\mathscr{F}_{f}^{T}$, it is necessary to have explicit formulas for the averaged discrete series characters. These formulas could in theory be obtained by summing the formulas given by Hirai in [11]. However, the formulas for the averaged discrete series for the classical infinite families $\left(B_{n}, C_{n}, D_{2 n}\right)$ having discrete series can be established independently of Hirai's general results. The simplicity of the averaged formulas in these important cases is not obvious from the general treatment in [9].

Thus the purpose of this paper is to establish the formulas for the averaged discrete series for the classical families of real simple Lie groups. These formulas will be used for work to appear on the Fourier inversion of stabilized invariant integrals and Plancherel theorem.

2. Averaged discrete series characters. We first establish some notation. For any reductive group $G$ and Cartan subgroup $H$, define $W(G, H)=N_{G}(H) / H$ where $N_{G}(H)$ is the normalizer of $H$ in $G$. Let $\Phi\left(\mathfrak{g}_{c}, \mathfrak{h}_{c}\right)$ denote the root system of the complexified Lie algebras of $G$ and $H$. Let $W(\Phi)$ denote the Weyl group of the root system $\Phi$. We regard $W(G, H)$ as a subgroup of $W\left(\Phi\left(\mathfrak{g}_{c}, \mathfrak{h}_{c}\right)\right)$. For $\lambda \in \mathfrak{h}_{c}^{*}$, we define $\xi_{\lambda}$ on $H_{c}$ by $\xi_{\lambda}(\exp H)=\exp (\lambda(H)), H \in \mathfrak{h}_{c}$, whenever this gives a well-defined character of $H_{c}$. Let $\Phi^{+}\left(\mathfrak{g}_{c}, \mathfrak{h}_{c}\right)$ denote a set of positive roots for $\Phi\left(\mathfrak{g}_{c}, \mathfrak{h}_{c}\right)$. Let $\delta=(1 / 2) \sum \alpha, \alpha \in \Phi^{+}\left(\mathfrak{g}_{c}, \mathfrak{h}_{c}\right)$. Then if $G$ is acceptable, $\xi_{\delta}$ is well-defined on $H$, and we define 


$$
\Delta(h)=\xi_{\delta}(h) \prod_{\alpha>0}\left(1-\xi_{\alpha}(h)^{-1}\right) .
$$

Let $H^{\prime}$ denote the set of regular elements in $H$; that is, $H^{\prime}=$ $\{h \in H \mid \Delta(h) \neq 0\}$.

Let $G$ be as in $\S 1$. Let $K$ be a maximal compact subgroup of $G$ containing $T$, and denote by $\theta$ the Cartan involution of $G$ with fixed point set $K$. Then $\mathfrak{g}$ has Cartan decomposition $\mathfrak{g}=\mathfrak{f}+\mathfrak{p}$ where $f$ is the Lie algebra of $K$. Let $H$ be a $\theta$-stable Cartan subgroup of $G$ with Lie algebra $\mathfrak{h}$. Write $\mathfrak{h}$ and $H$ according to their Cartan decompositions as $\mathfrak{h}=\mathfrak{h}_{1}+\mathfrak{h}_{p}$ and $H=H_{K} H_{p}$. Let $y \in G_{C}$ satisfy $\operatorname{ady}\left(\mathfrak{t}_{c}\right)=\mathfrak{h}_{c}$. Then $y$ induces a mapping from $\mathfrak{t}_{c}^{*}$ to $\mathfrak{h}_{c}^{*}$ which we denote by $\tau \rightarrow{ }^{y} \tau$.

Let $h \in H^{\prime}$. Write $h=h_{K} h_{p}$ where $h_{K} \in H_{K}, h_{p} \in H_{p}$. Let $H_{K}$ be the connected component of $H_{K}$ containing $h_{K}$. Assume $H_{K}^{\llcorner} \cong T$. Let $z$ denote the centralizer in $g$ of $H_{K}^{+}, Z$ the connected subgroup of $G$ with Lie algebra $z$. Let $\Phi=\Phi\left(z_{c}, \mathrm{t}_{c}\right)$. We consider $\Phi$ as a subset of $\Phi\left(\mathrm{g}_{c}, \mathrm{t}_{c}\right)$. Let $\Phi^{+}=\left\{\alpha \in \Phi \mid{ }^{y} \alpha\left(\log h_{p}\right)>0\right\}$. Let $\tau \in L_{r}$ and denote by $\theta_{\tau}$ the corresponding invariant eigendistribution defined by HarishChandra. Then it follows from [2] that:

$$
\theta_{\tau}\left(h_{K} h_{p}\right)=\Delta(h)^{-1} \sum_{t \in W(Z, T) \backslash W(G, T)} \operatorname{det} t \sum_{S \in W(\phi)} \operatorname{det} s c\left(s: t \tau: \Phi^{+}\right) \xi_{s t \tau}\left(y^{-1} h\right) .
$$

The $c\left(s: \tau: \Phi^{+}\right)$are integers satisfying:

$$
c\left(s u: \tau: \Phi^{+}\right)=c\left(s: u \tau: \Phi^{+}\right), \quad u \in W(Z, T) .
$$

Lemma 2.3. Let $W=W\left(\mathfrak{g}_{c}, \mathfrak{t}_{c}\right)$, other notation as above. Then

$$
\sum_{w \in W} \operatorname{det} w \theta_{w \tau}(h)=[W(G, T)] \Delta(h)^{-1} \sum_{w \in W} \operatorname{det} w \bar{c}\left(w \tau: \Phi^{+}\right) \xi_{w \tau}\left(y^{-1} h\right)
$$

where $\bar{c}\left(\tau: \Phi^{+}\right)=\sum_{s \in W(\Phi) \backslash W(Z, T)} c\left(s: s^{-1} \tau: \Phi^{+}\right)$.

Proof. The formula follows directly from (2.1) and (2.2) since $W(G, T)$ and $W(\Phi)$ can both be regarded as subgroups of $W$.

The constants $\bar{c}\left(\tau: \Phi^{+}\right)$have the following properties which can be deduced from their definition and from the corresponding properties of the constants $c\left(s: \tau: \Phi^{+}\right)$proved in [2].

$$
\bar{c}\left(s \tau: s \Phi^{+}\right)=\bar{c}\left(\tau: \Phi^{+}\right) \quad \text { for } \quad s \in W(\Phi) .
$$

Let $\left\{\alpha_{1}, \cdots, \alpha_{l}\right\}$ be a set of simple roots for $\Phi^{+}$. Let $A_{1}, \cdots, A_{l}$ in $L_{T}$ satisfy $\left\langle\Lambda_{i}, \alpha_{j}\right\rangle=\delta_{i j}$. Then:

$$
\bar{c}\left(\tau: \Phi^{+}\right)=0 \text { if }\left\langle\tau, \Lambda_{i}\right\rangle>0 \text { for any } 1 \leqq i \leqq l .
$$


Let $\alpha \in \Phi^{+}$be a simple root. Let $\alpha_{0}={ }^{y} \alpha \in \Phi\left(\mathfrak{z}_{c}, \mathfrak{h}_{c}\right)$. Let $X_{\alpha_{0}}$ and $Y_{\alpha_{0}}$ denote the root vectors for $\alpha_{0}$ and $-\alpha_{0}$ in $\mathrm{g}$ satisfying $\left[X_{\alpha_{0}}, Y_{\alpha_{0}}\right]=$ $2 H_{\alpha 0} /\left\langle\alpha_{0}, \alpha_{0}\right\rangle$ where $H_{\alpha_{0}} \in \mathfrak{h}$ satisfies $\beta\left(H_{\alpha_{0}}\right)=\left\langle\beta, \alpha_{0}\right\rangle$ for all $\beta \in \Phi\left(\mathfrak{g}_{c}, \mathfrak{h}_{c}\right)$. Let $\nu=\exp \left((-\pi \sqrt{-1(1) / 4})\right.$ ad $\left.\left(X_{\alpha_{0}}+Y_{\alpha_{0}}\right)\right)$. Then $\mathfrak{i}=\nu\left(\mathfrak{h}_{c}\right) \cap \mathfrak{g}$ is a $\theta$ stable Cartan subalgebra of g. Let $\Phi_{0}=\{\beta \in \Phi \mid\langle\beta, \alpha\rangle=0\}, \Phi_{0}^{+}=\Phi_{0} \cap \Phi^{+}$. Let $s$ denote the reflection in $W(\Phi)$ corresponding to $\alpha$. Then:

$$
\bar{c}\left(\tau: \Phi^{+}\right)+\bar{c}\left(s \tau: \Phi^{+}\right)=\bar{c}\left(\tau: \Phi_{0}^{+}\right)+\bar{c}\left(s \tau: \Phi_{0}^{+}\right)=2 \bar{c}\left(\tau ; \Phi_{0}^{+}\right)
$$

since $\bar{c}\left(s \tau: \Phi_{0}^{+}\right)=\bar{c}\left(\tau: s \Phi_{0}^{+}\right)=\bar{c}\left(\tau: \Phi_{0}^{+}\right)$as $s \beta=\beta$ for all $\beta \in \Phi_{0}$.

Let $\mathscr{F}$ be the real subspace of $\sqrt{-1}$ t spanned by $\Phi$. For $\tau \in$ $\sqrt{-1} t^{*}, \tau$ can be written uniquely as $\tau=\tau_{I}+\tau_{0}$, where $\tau_{0} \in \mathscr{F}$, and ${ }^{y} \tau_{I}$ takes purely imaginary values of $\mathfrak{h}$. Let $\mathscr{F}^{\prime}=\{\lambda \in \mathscr{F} \mid\langle\lambda, \alpha\rangle \neq$ $0, \alpha \in \Phi\}$. Then $\bar{c}\left(\tau: \Phi^{+}\right)$depends only on the component $\mathscr{F}^{+}$of $\tau_{0}$ in $\mathscr{F}^{\prime}$. We write

$$
\bar{c}\left(\mathscr{F}^{+}: \Phi^{+}\right)=\bar{c}\left(\tau: \Phi^{+}\right) \quad \text { if } \quad \tau_{0} \in \mathscr{T}^{+} .
$$

If $\Phi=\Phi_{1} \cup \cdots \cup \Phi_{s}$ where the $\Phi_{i}, 1 \leqq i \leqq s$, are simple root systems, then $\lambda \in \mathscr{F}^{+}$can be written uniquely as $\lambda=\lambda_{1}+\cdots+\lambda_{s}$ where for $1 \leqq i \leqq s, \lambda_{i} \in \mathscr{F}_{i}$, the real linear span of the elements of $\Phi_{i}$. Let $\mathscr{F}_{i}{ }^{+}$be the component of $\mathscr{F}_{i}{ }^{\prime}=\left\{\lambda \in \mathscr{F}_{i} \mid\langle\alpha, \lambda\rangle \neq 0, \alpha \in \Phi_{i}\right\}$ containing $\lambda_{i}$. Then if $\Phi_{i}^{+}=\Phi_{i} \cap \Phi^{+}$,

$$
\bar{c}\left(\mathscr{F}^{+}: \Phi^{+}\right)=\prod_{i=1}^{s} \bar{c}\left(\mathscr{F}_{i}^{+}: \Phi_{i}^{+}\right) .
$$

Note that if $\Phi\left(\mathfrak{g}_{c}, \mathrm{t}_{c}\right)$ is of classical type, so are all the simple components $\Phi_{i}$ in the decomposition of $\Phi$.

We see that the problem of computing constants for averaged discrete series characters reduces to the problem of computing certain constants $\bar{c}\left(\mathscr{F}^{+}: \Phi^{+}\right)$connected to a simple root system $\Phi$, a choice of positive roots $\Phi^{+}$, and a component $\mathscr{F}^{+} \subseteq \mathscr{F}^{\prime}$, the set of regular elements in the underlying real vector space of $\Phi$. We will derive formulas for these constants for the cases $\Phi=B_{n}, C_{n}$, or $D_{n}, n \geqq 1$, where for $D_{n}$ we assume $n$ is even. (Of course, $B_{1}=C_{1}=A_{1}$, and $D_{2}=A_{1}^{2}$ )

Let

$$
\Phi=\left\{\begin{array}{l}
\left\{ \pm e_{i} \pm e_{j}, \pm e_{i} \mid 1 \leqq i \neq j \leqq n\right\} \text { if } \Phi=B_{n} \\
\left\{ \pm e_{i} \pm e_{j}, \pm 2 e_{i} \mid 1 \leqq i \neq j \leqq n\right\} \text { if } \Phi=C_{n} \\
\left\{ \pm e_{i} \pm e_{j} \mid 1 \leqq i \neq j \leqq n\right\} \text { if } \Phi=D_{n} .
\end{array}\right.
$$

Assume

$$
\Phi^{+}=\left\{\begin{array}{l}
\left\{e_{i} \pm e_{j}, e_{K} \mid 1 \leqq i<j \leqq n, 1 \leqq K \leqq n\right\} \text { if } \Phi=B_{n}, \\
\left\{e_{i} \pm e_{j}, 2 e_{K} \mid 1 \leqq i<j \leqq n, 1 \leqq K \leqq n\right\} \text { if } \Phi=C_{n}, \\
\left\{e_{i} \pm e_{j} \mid 1 \leqq i<j \leqq n\right\} \text { if } \Phi=D_{n} .
\end{array}\right.
$$


Then a set $S$ of simple roots for $\Phi^{+}$is given by:

$$
S=\left\{\begin{array}{l}
\left\{e_{1}-e_{2}, e_{2}-e_{3}, \cdots, e_{n-1}-e_{n}, e_{n}\right\} \text { if } \Phi=B_{n}, \\
\left\{e_{1}-e_{2}, e_{2}-e_{3}, \cdots, e_{n-1}-e_{n}, 2 e_{n}\right\} \text { if } \Phi=C_{n}, \\
\left\{e_{1}-e_{2}, e_{2}-e_{3}, \cdots, e_{n-1}-e_{n}, e_{n-1}+e_{n}\right\} \text { if } \Phi=D_{n} .
\end{array}\right.
$$

In each case an element $\lambda$ of $\mathscr{F}$ can be written as $\lambda=\sum_{i=1}^{n} m_{i} e_{i}$, $m_{i} \in \boldsymbol{R}$. (If $\lambda$ is in the weight lattice for $\Phi$, the $m_{i}$ 's will be integers or half-integers.) In each case the permutation group $S_{n}$ on $n$ elements acts on $\Phi$ and on $\mathscr{F}$ by permuting the indices of the $e_{i}$ 's. With this action, $S_{n}$ is a subgroup of $W(\Phi)$. Let $S_{n}^{*}=$ $\left\{\sigma \in S_{n} \mid \sigma(2 i-1)<\sigma(2 i), 1 \leqq i \leqq[n / 2]\right\}$ and $S_{n}^{* *}=\left\{\sigma \in S_{n}^{*} \mid \sigma(1)<\sigma(3)<\right.$ $\cdots<\sigma(2[n / 2]-1)\}$. For $\lambda=\sum_{i=1}^{n} m_{i} e_{i}$, let $\lambda_{i}=m_{2 i-1} e_{1}+m_{2 i} e_{2}, 1 \leqq$ $i \leqq[n / 2]$. If $n$ is odd, let $\lambda_{n}=m_{n} e_{1}$. If $\Phi$ is of type $B_{n}$ or $C_{n}$, let

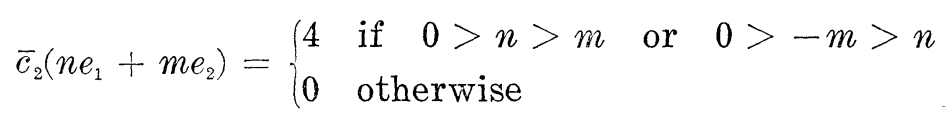

$$
\bar{c}_{1}\left(n e_{1}\right)=\left\{\begin{array}{lll}
2 & \text { if } & n<0 \\
0 & \text { if } & n>0
\end{array} .\right.
$$

If $\Phi$ is of type $D_{n}$, let

$$
\bar{c}_{2}\left(n e_{1}+m e_{2}\right)= \begin{cases}4 & \text { if } n<-|m| \\ 0 & \text { otherwise }\end{cases}
$$

Theorem 2.12. If $\lambda \in \mathscr{F}^{+}$, then $\bar{c}\left(\mathscr{F}^{+}: \Phi^{+}\right)=P\left(\lambda: \Phi^{+}\right)$where

$$
P\left(\lambda: \Phi^{+}\right)=\sum_{\sigma \in S_{n}^{* * *}} \operatorname{det} \sigma \prod_{i=1}^{n / 2} \bar{c}_{2}\left(\left(\sigma^{-1} \lambda\right)_{i}\right)
$$

if $n$ is even

$$
=\sum_{\sigma \in S_{n}^{* *}} \operatorname{det} \sigma \bar{c}_{1}\left(\left(\sigma^{-1} \lambda\right)_{n}\right) \prod_{i=1}^{n-1 / 2} \bar{c}_{2}\left(\left(\sigma^{-1} \lambda\right)_{i}\right)
$$

if $n$ is odd.

Proof. The theorem is true for $n=1$ or 2 because it reduces to formulas (2.9), (2.10), and (2.11) which are known from averaging the known discrete series constants for rank one and two groups $[3,7]$. Assume that it is true for root systems of rank less than $n, n \geqq 3$. We prove in Lemma 2.14 that for any simple root $\alpha$, $P\left(\lambda: \Phi^{+}\right)+P\left(s \lambda: \Phi^{+}\right)=2 \bar{c}\left(\lambda: \Phi_{0}^{+}\right)$where $s$ is the reflection in $W(\Phi)$ corresponding to $\alpha$ and $\Phi_{0}=\{\beta \in \Phi \mid\langle\beta, \alpha\rangle=0\}$ as in (2.6). Then using (2.6), $P\left(\lambda_{:}: \Phi^{+}\right)+P\left(s \lambda: \Phi^{+}\right)=\bar{c}\left(\mathscr{F}{ }^{+}: \Phi^{+}\right)+\bar{c}\left(s \mathscr{F}{ }^{+}: \Phi^{+}\right)$for $\lambda \in \mathscr{F}^{+}$. We show in Lemma 2.13 that $\bar{c}\left(\mathscr{F}^{*}: \Phi^{+}\right)=P\left(\lambda: \Phi^{+}\right), \lambda \in \mathscr{F}^{\sharp}$, for one 
fixed chamber $\mathscr{F}^{\sharp}$ of $\mathscr{F}^{\prime}$. Then $\bar{c}\left(\mathscr{F}^{+}: \Phi^{+}\right)=P\left(\lambda: \Phi^{+}\right), \lambda \in \mathscr{F}^{+}$, for all chambers, since any chamber $\mathscr{F}^{+}$can be reached by applying simple reflections to $\mathscr{F}^{\sharp}$.

Lemma 2.13. Let $\mathscr{F}^{+}=\left\{\lambda \in \mathscr{F}^{-} \mid\langle\lambda, \alpha\rangle>0\right.$ for all $\left.\alpha \in \Phi^{+}\right\}$. Then $\bar{c}\left(\mathscr{F}^{-}: \Phi^{+}\right)=P\left(\lambda: \Phi^{+}\right)$if $\lambda \in \mathscr{F}^{+}$.

Proof. It follows from (2.5) that $\bar{c}\left(\mathscr{F}^{\sharp}: \Phi^{+}\right)=0$. For $\lambda=$ $\sum_{i=1}^{n} m_{i} e_{i}, \lambda \in \mathscr{F}^{*}$ implies that $m_{i}>\left|m_{j}\right|$ for $1 \leqq i<j \leqq n$. For any $\sigma \in S_{n}^{* *}$ and any $1 \leqq i \leqq[n / 2],\left(\sigma^{-1} \lambda\right)_{i}=m_{\sigma(2 i-1)} e_{1}+m_{\sigma(2 i)} e_{2}$ where $\sigma(2 i-1)<\sigma(2 i)$. Thus $m_{\sigma(2 i-1)}>\left|m_{\sigma(2 i)}\right|$ and using (2.9) or (2.11), $\bar{c}_{2}\left(\left(\sigma^{-1} \lambda\right)_{i}\right)=0$. Thus $P\left(\lambda: \Phi^{+}\right)=0$.

LeMma 2.14. Assume that Theorem 2.12 is true for root systems of rank less then $n$. Let $\alpha$ be a simple root for $\Phi^{+}$. Then for $\lambda \in \mathscr{T}^{+}, P\left(\lambda: \Phi^{+}\right)+P\left(s \lambda: \Phi^{+}\right)=2 \bar{c}\left(\lambda: \Phi_{0}^{+}\right)$.

\section{Proof.}

Case $I$. Suppose $\alpha=e_{l}-e_{t+1}, 1 \leqq l \leqq n-1$. Let $\Phi_{n-2}$ denote the subset of $\Phi$ contained in the linear span of $\left\{e_{1}, \cdots, e_{l-1}, e_{l+2}, \cdots, e_{n}\right\}$, $\Phi_{n-2}^{+}=\Phi_{n-2} \cap \Phi^{+}$. Let $A_{1}$ denote the rank one root system with positive root $e_{l}+e_{i+1}$. Then $\Phi_{0}^{+}=\Phi_{n-2}^{+} \cup A_{1}^{+}$. For $\lambda=\sum_{i=1}^{n} m_{i} e_{i}$, let $\lambda^{\prime}=\lambda-m_{l} e_{l}-m_{l+1} e_{l+1}$ and $\lambda^{\prime \prime}=\left(m_{l}+m_{l+1}\right) / 2\left(e_{l}+e_{l+1}\right)$. Let $\lambda_{0}=$ $\lambda^{\prime}+\lambda^{\prime \prime}$. Then by (2.8),

$$
\bar{c}\left(\lambda: \Phi_{0}^{+}\right)=\bar{c}\left(\lambda^{\prime \prime}: A_{1}^{+}\right) \bar{c}\left(\lambda^{\prime}: \Phi_{n-2}^{+}\right)=\bar{c}_{1}\left(\left(m_{l}+m_{l+1}\right) e_{1}\right) P\left(\lambda^{\prime}: \Phi_{n-2}^{+}\right)
$$

by the induction hypothesis. In $P\left(\lambda^{\prime}: \Phi_{n-2}^{+}\right)$the sum is taken over $S_{n-2}^{* *}$ where $S_{n-2}$ is considered as the group of permutations of $\{1,2, \cdots, l-1, l+2, \cdots, n\}$ and $\left(\sigma^{-1} \lambda^{\prime}\right)_{i}, 1 \leqq i \leqq[(n-2) / 2]$ and $\left(\sigma^{-1} \lambda^{\prime}\right)_{n-2}, n-2$ odd, have the obvious meaning.

Let $k=[n / 2]$ so that $n=2 k$ or $2 k+1$. In formulas for $P\left(\lambda: \Phi^{+}\right)$ the terms $\bar{c}_{1}\left(\left(\sigma^{-1} \lambda\right)_{n}\right)$ are included. For the case $n=2 k$ they are understood not to appear. If $s$ is the reflection in $W(\Phi)$ corresponding to $\alpha=e_{l}-e_{i+1}$, then $s$ is the permutation which interchanges $l$ and $\boldsymbol{l}+1$. $P\left(\lambda: \Phi^{+}\right)+P\left(s \lambda: \Phi^{+}\right)=1 / k ! \sum_{\sigma \in S_{n}^{*}} \operatorname{det} \sigma\left[\bar{c}_{1}\left(\left(\sigma^{-1} \lambda\right)_{n}\right) \prod_{i=1}^{k} \bar{c}_{2}\left(\left(\sigma^{-1} \lambda\right)_{i}\right)+\right.$ $\left.\bar{c}_{1}\left(\left((s \sigma)^{-1} \lambda\right)_{n}\right) \prod_{i=1}^{k} \bar{c}_{2}\left(\left((s \sigma)^{-1} \lambda\right)_{i}\right)\right]$.

Let $S=\left\{\sigma \in S_{n}^{*} \mid s \sigma \in S_{n}^{*}\right\}$. Then $s S=S$, and

$\sum_{\sigma \in S} \operatorname{det} \sigma \bar{c}_{1}\left(\left((s \sigma)^{-1} \lambda\right)_{n}\right) \prod_{i=1}^{k} \bar{c}_{2}\left(\left((s \sigma)^{-1} \lambda\right)_{i}\right)=-\sum_{\sigma \in S} \operatorname{det} \sigma \bar{c}_{1}\left(\left(\sigma^{-1} \lambda\right)_{n}\right) \prod_{i=1}^{k} \bar{c}_{2}\left(\left(\sigma^{-1} \lambda\right)_{i}\right)$.

If $\sigma \in S_{n}^{*}$ and $s \sigma \notin S_{n}^{*}$, then there is an index $j, 1 \leqq j \leqq k$, for which $\sigma(2 j-1)=\boldsymbol{l}, \sigma(2 j)=\boldsymbol{l}+1$. Denote this subset of $S_{n}^{*}$ by $S(j)$. Then for $\sigma \in S(j), s \sigma(2 j-1)=l+1, s \sigma(2 j)=l$, and for $i \neq 2 j-1,2 j$, 
$s \sigma(i)=\sigma(i)$. Further, using (2.9), (2.10), and (2.11), $\bar{c}_{2}\left(\left(\sigma^{-1} \lambda\right)_{j}\right)+$ $\bar{c}_{2}\left(\left((s \sigma)^{-1} \lambda\right)_{j}\right)=\bar{c}_{2}\left(m_{l} e_{1}+m_{l+1} e_{2}\right)+\bar{c}_{2}\left(m_{l+1} e_{1}+m_{l} e_{2}\right)=2 \bar{c}_{1}\left(\left(m_{l}+m_{l+1}\right) e_{1}\right)$. Thus

$$
\begin{aligned}
P\left(\lambda: \Phi^{+}\right)+P\left(s \lambda: \Phi^{+}\right)= & \frac{1}{k !} \sum_{j=1}^{k} \sum_{\sigma \in S(j)} \operatorname{det} \sigma \bar{c}_{1}\left(\left(\sigma^{-1} \lambda\right)_{n}\right) \\
& \times \prod_{\substack{i=1 \\
i \neq j}}^{k} \bar{c}_{2}\left(\left(\sigma^{-1} \lambda\right)_{i}\right) 2 \bar{c}_{1}\left(\left(m_{l}+m_{\boldsymbol{l}+1}\right) e_{1}\right) .
\end{aligned}
$$

For each $1 \leqq j \leqq k$,

$$
\begin{aligned}
\sum_{\sigma \in S(j)} \operatorname{det} \sigma \bar{c}_{1}\left(\left(\sigma^{-1} \lambda\right)_{n}\right) \prod_{\substack{i=1 \\
i \neq j}}^{k} \bar{c}_{2}\left(\left(\sigma^{-1} \lambda\right)_{i}\right) \\
=\sum_{\sigma \in S_{n-2}^{*}} \operatorname{det} \sigma \bar{c}_{1}\left(\left(\sigma^{-1} \lambda^{\prime}\right)_{n-2}\right) \prod_{i=1}^{k-1} \bar{c}_{2}\left(\left(\sigma^{-1} \lambda^{\prime}\right)_{i}\right)=(k-1) ! \bar{c}\left(\lambda^{\prime}: \Phi_{n-2}^{+}\right) .
\end{aligned}
$$

Thus

$$
P\left(\lambda: \Phi^{+}\right)+P\left(s \lambda: \Phi^{+}\right)=2 \bar{c}_{1}\left(\left(m_{l}+m_{l+1}\right) e_{1}\right) \bar{c}\left(\lambda^{\prime}: \Phi_{n-2}^{+}\right)=2 \bar{c}\left(\lambda_{0}: \Phi_{0}^{+}\right) .
$$

Case II. Suppose $\Phi=B_{n}$ or $C_{n}$ and $\alpha=e_{n}$ or $2 e_{n}$. Then $\Phi_{0}=B_{n-1}$ or $C_{n-1}$. For $\lambda=\sum_{i=1}^{n} m_{i} e_{i}$, let $\lambda_{0}=\lambda-m_{n} e_{n}$. By the induction hypothesis, $\bar{c}\left(\lambda_{0}: \Phi_{0}^{+}\right)=P\left(\lambda_{0}: \Phi_{0}^{+}\right)$.

Suppose $n=2 k$ is even. Then $S_{n}^{*}=\bigcup_{j=1}^{k} S(j)$ where $S(j)=$ $\left\{\sigma \in S_{n}^{*} \mid \sigma(2 j)=n\right\}$. For $\sigma \in S(j),\left(\sigma^{-1} \lambda\right)_{i}=\left(\sigma^{-1} s \lambda\right)_{i}$ for $i \neq j,\left(\sigma^{-1} \lambda\right)_{j}=$ $m_{\sigma(2 j-1)} e_{1}+m_{n} e_{2}$, and $\left(\sigma^{-1} s \lambda\right)_{j}=m_{\sigma(2 j-1)} e_{1}-m_{n} e_{2}$. Using (2.9) and (2.10), $\bar{c}_{2}\left(m_{\sigma(2 j-1)} e_{1}+m_{n} e_{2}\right)+\bar{c}_{2}\left(m_{\sigma(2 j-1)} e_{1}-m_{n} e_{2}\right)=2 \bar{c}_{1}\left(m_{\sigma(2 j-1)} e_{1}\right)$.

$$
\begin{aligned}
P(\lambda: & \left.\Phi^{+}\right)+P\left(s \lambda: \Phi^{+}\right) \\
& =\frac{1}{k !} \sum_{j=1}^{k} \sum_{\sigma \in S(j)} \operatorname{det} \sigma 2 \bar{c}_{1}\left(m_{\sigma(2 j-1)} e_{1}\right) \prod_{\substack{i=1 \\
i \neq j}}^{k} \bar{c}_{2}\left(\left(\sigma^{-1} \lambda\right)_{i}\right) \\
& =\frac{2}{(k-1) !} \sum_{\sigma \in S_{n-1}^{*}} \operatorname{det} \sigma \bar{c}_{1}\left(m_{\sigma(n-1)} e_{1}\right) \prod_{i=1}^{k-1} \bar{c}_{2}\left(\left(\sigma^{-1} \lambda\right)_{i}\right)=2 \bar{c}\left(\lambda_{0}: \Phi_{0}^{+}\right) .
\end{aligned}
$$

Suppose $n=2 k+1$. Define $S(j)$ as above. Then $S_{n}^{*}=\bigcup_{j=1}^{n} S(j) \cup$ $S_{n-1}^{*} \quad$ where $S_{n-1}^{*}$ can be identified with $\left\{\sigma \in S_{n}^{*} \mid \sigma(n)=n\right\}$. For $\sigma \in S_{n-1}^{*},\left(\sigma^{-1} \lambda\right)_{i}=\left(\sigma^{-1} s \lambda\right)_{i}, 1 \leqq i \leqq k, \quad$ and $\quad \bar{c}_{1}\left(\left(\sigma^{-1} \lambda\right)_{n}\right)+\bar{c}_{1}\left(\left(\sigma^{-1} s \lambda\right)_{n}\right)=$ $\bar{c}_{1}\left(m_{n} e_{1}\right)+\bar{c}_{1}\left(-m_{n} e_{1}\right)=2$, using (2.10).

$$
\begin{aligned}
P\left(\lambda: \Phi^{+}\right) & +P\left(s \lambda: \Phi^{+}\right) \\
= & \frac{1}{k !} \sum_{j=1}^{k} \sum_{\sigma \in S(j)} \operatorname{det} \sigma 2 \bar{c}_{1}\left(m_{\sigma(2 j-1)} e_{1}\right) \bar{c}_{1}\left(m_{\sigma(n)} e_{1}\right) \prod_{\substack{i=1 \\
i \neq j}}^{k} \bar{c}_{2}\left(\left(\sigma^{-1} \lambda\right)_{i}\right) \\
& +\frac{2}{k !} \sum_{\sigma \in S_{n-1}^{*}} \operatorname{det} \sigma \prod_{i=1}^{k} \bar{c}_{2}\left(\left(\sigma^{-1} \lambda\right)_{i}\right) .
\end{aligned}
$$


The second term in (2.15) involving the sum over $S_{n-1}^{*}$ is exactly $2 \bar{c}\left(\lambda_{0}: \Phi_{0}^{+}\right)$. For each $1 \leqq j \leqq k$, let $S(j)^{+}=\{\sigma \in S(j) \mid \sigma(2 j-1)<\sigma(n)\}$. Let $S(j)^{-}=\{\sigma \in S(j) \mid \sigma(2 j-1)>\sigma(n)\}$. Let $\tau$ denote the permutation $(2 j-1 n)$ which interchanges $2 j-1$ and $n$. Then $S(j)^{-}=\left\{\sigma \tau \mid \sigma \in S(j)^{+}\right\}$ and

$$
\begin{aligned}
\sum_{\sigma \in S(j)-} \operatorname{det} \sigma \bar{c}_{1}\left(m_{\sigma(2 j-1} e_{1}\right) \bar{c}_{1}\left(m_{\sigma(n)} e_{1}\right) \prod_{\substack{i=1 \\
i \neq j}}^{k} \bar{c}_{2}\left(\left(\sigma^{-1} \lambda\right)_{i}\right) \\
=-\sum_{\sigma \in S(j)+-}+\operatorname{det} \sigma \bar{c}_{1}\left(m_{\sigma(n)} e_{1}\right) \bar{c}_{1}\left(m_{\sigma(2 j-1)} e_{1}\right) \prod_{\substack{i=1 \\
i \neq j}}^{k} \bar{c}_{2}\left(\left(\sigma^{-1} \lambda\right)_{i}\right) .
\end{aligned}
$$

Thus the sum over $S(j)$ in (2.15) is zero for each $j$, and $P\left(\lambda: \Phi^{+}\right)+$ $P\left(s \lambda: \Phi^{+}\right)=2 \bar{c}\left(\lambda_{0}: \Phi^{+}\right)$.

Case III. Suppose $\Phi=D_{n}$ where $n=2 k$ is even, and $\alpha=e_{n-1}+e_{n}$. Then $\Phi_{0}^{+}=D_{n-2}^{+} \cup A_{1}^{+}$where $A_{1}$ has positive root $e_{n-1}-e_{n}$. For $\lambda=$ $\sum_{i=1}^{n} m_{i} e_{i}$, let $\lambda^{\prime}=\lambda-m_{n-1} e_{n-1}-m_{n} e_{n}$ and $\lambda^{\prime \prime}=\left(m_{n-1}-m_{n} / 2\right)\left(e_{n-1}-e_{n}\right)$. Let $\lambda_{0}=\lambda^{\prime}+\lambda^{\prime \prime}$. For $1 \leqq j \leqq k$, let $S(j)=\left\{\sigma \in S_{n}^{*} \mid \sigma(2 j-1)=n-1\right.$, $\sigma(2 j)=n\}$. Note $S(k)=S_{n-2}^{*}$. For $1 \leqq l \neq j \leqq k$, let $S(l, j)=$ $\left\{\sigma \in S_{n}^{*} \mid \sigma(2 \boldsymbol{l})=n-1, \sigma(2 j)=n\right\}$. Then $S_{n}^{*}=\bigcup_{j=1}^{k} S(j) \cup \bigcup_{1 \leq l \neq j \leq l} S(\boldsymbol{l}, j)$. For $\sigma \in S(j),\left(\sigma^{-1} \lambda\right)_{i}=\left(\sigma^{-1} s \lambda\right)_{i}, i \neq j$, and $\bar{c}_{2}\left(\left(\sigma^{-1} \lambda\right)_{j}\right)+\bar{c}_{2}\left(\left(\sigma^{-1} s \lambda\right)_{j}\right)=$ $\bar{c}_{2}\left(m_{n-1} e_{1}+m_{n} e_{2}\right)+\bar{c}_{2}\left(-m_{n} e_{1}-m_{n-1} e_{2}\right)=2 \bar{c}_{1}\left(\left(m_{n-1}-m_{n}\right) e_{1}\right)$ using (2.10) and (2.11). For $\sigma \in S(l, j),\left(\sigma^{-1} \lambda\right)_{i}=\left(\sigma^{-1} s \lambda\right)_{i}, i \neq j$ or $\boldsymbol{l}$, and

$$
\begin{aligned}
\bar{c}_{2}\left(\left(\sigma^{-1} s \lambda\right)_{j}\right) \bar{c}_{2}\left(\left(\sigma^{-1} s \lambda\right)_{\imath}\right) & =\bar{c}_{2}\left(m_{\sigma(2 j-1)} e_{1}-m_{n-1} e_{2}\right) \bar{c}_{2}\left(m_{\sigma(2 l-1)} e_{1}-m_{n} e_{2}\right) \\
& =\bar{c}_{2}\left(m_{\sigma(2 j-1)} e_{1}+m_{n-1} e_{2}\right) \cdot \bar{c}_{2}\left(m_{\sigma(2 l-1)} e_{1}+m_{n} e_{2}\right)
\end{aligned}
$$

using (2.11).

Then

$$
\begin{aligned}
P\left(\lambda: \Phi^{+}\right) & +P\left(s \lambda: \Phi^{+}\right) \\
= & \frac{1}{k !} \sum_{j=1}^{k} \sum_{\sigma \in S(j)} \operatorname{det} \sigma \prod_{\substack{i=1 \\
i \neq j}}^{k} \bar{c}_{2}\left(\left(\sigma^{-1} \lambda\right)_{i}\right) 2 \bar{c}_{1}\left(\left(m_{n-1}-m_{n}\right) e_{1}\right) \\
& +\frac{1}{k !} \sum_{1 \leqq j \neq i \leqq l} \sum_{\sigma \in S(l, j)} \operatorname{det} \sigma \prod_{\substack{i=1 \\
i \neq j, l}}^{k} \bar{c}_{2}\left(\left(\sigma^{-1} \lambda\right)_{i}\right) \\
& \times\left[\bar{c}_{2}\left(m_{\sigma(2 j-1)} e_{1}+m_{n-1} e_{2}\right) \bar{c}_{2}\left(m_{\sigma(2 l-1)} e_{1}+m_{n} e_{2}\right)\right. \\
& \left.+\bar{c}_{2}\left(m_{\sigma(2 j-1)} e_{1}+m_{n} e_{2}\right) \bar{c}_{2}\left(m_{\sigma(2 l-1)} e_{1}+m_{n-1} e_{2}\right)\right] .
\end{aligned}
$$

For each

$$
\begin{aligned}
1 & \leqq j \leqq k, \sum_{\sigma \in S(j)} \operatorname{det} \sigma \prod_{\substack{i=1 \\
i \neq j}}^{k} \bar{c}_{2}\left(\left(\sigma^{-1} \lambda\right)_{i}\right) \\
& =\sum_{\sigma \in S_{n-2}^{*}} \operatorname{det} \sigma \prod_{i=1}^{k-1} \bar{c}_{2}\left(\left(\sigma^{-1} \lambda\right)_{i}\right)=(k-1) ! \bar{c}\left(\lambda^{\prime}: D_{n-2}^{+}\right) .
\end{aligned}
$$


For $1 \leqq j \neq l \leqq k$, let $S(l, j)^{+}=\{\sigma \in S(l, j) \mid \sigma(2 \boldsymbol{l}-1)<\sigma(2 j-1)\}$ and $S(l, j)^{-}=\{\sigma \in S(l, j) \mid \sigma(2 l-1)>\sigma(2 j-1)\}$. Then $S(l, j)^{-}=$ $\left\{\sigma \tau \mid \sigma \in S(l, j)^{+}\right\}$where $\tau=(2 j-1,2 \boldsymbol{l}-1)$. Then the sum over $S(l, j)$ in (2.16) is zero since the sum over $S(l, j)^{-}$will cancel with the sum over $S(l, j)^{+}$for each $l$ and $j$. Thus

$$
P\left(\lambda: \Phi^{+}\right)+P\left(s \lambda ! \Phi^{+}\right)=2 \bar{c}_{1}\left(\left(m_{n-1}-m_{n}\right) e_{1}\right) \bar{c}\left(\lambda^{\prime}: D_{n-2}^{+}\right)=2 \bar{c}\left(\lambda_{0}: \Phi_{0}^{+}\right) .
$$

\section{REFERENCES}

1. Harish-Chandra, A formula for semisimple Lie groups, Amer. J. Math., 79 (1957), 733-760.

2. - Discrete series for semisimple Lie groups I, Acta Math., 113 (1965), 241-318.

3. - Two theorems on semisimple Lie groups, Annals of Math., (2) 83 (1966), 74-128.

4. H. Hecht, The characters of Harish-Chandra representations, to appear in Math. Annalen.

5. R. Herb, Character formulas for discrete series on semisimple Lie groups, Nagoya Math. J., 64 (1976), 47-61.

6. T. Hirai, Invariant eigendistributions of Laplace operators on real simple Lie groups I, case of $S U(p, q)$, Japan J. Math., 39 (1970), 1-68.

7. - Explicit form of the characters of discrete series representations of semisimple Lie groups, Proceedings of Symposia in Pure Math., 26, 281-287, Amer. Math. Soc., 1973.

8. - Invariant eigendistributions of Laplace operators on real simple Lie groups IV, Characters of discrete series for $S p(n, \mathbf{R})$, Japan J. Math., 3 (1977), 1-48.

9. — The characters of the discrete series for semisimple Lie groups, to appear. 10. S. Martens, The characters of the holomorphic discrete series, Proc. Nat. Acad. Sci. USA, 72 (1975), 3275-3276.

11. H. Midorikawa, On the explicit formulae of characters for discrete series representations, to appear in J. Math. Soc., Japan.

12. W. Schmid, On the characters of the discrete series, Inventiones Math., 30 (1975), $47-144$.

Received March 6, 1978

UNIVERSITY OF MARYLAND

COLLEGE PARK, MD 20742 



\section{PACIFIC JOURNAL OF MATHEMATICS}

\section{EDITORS}

RICHARD ARENS (Managing Editor)

University of California

Los Angeles, California 90024

C. W. CURTIS

University of Oregon

Eugene, OR 97403

C. C. MOORE

University of California

Berkeley, CA 94720
J. DugundjI

Department of Mathematics University of Southern Californı Los Angeles, California 90007

R. Finn and J. Milgram Stanford University Stanford, California 94305

ASSOCIATE EDITORS

E. F. BECKENBACH

B. H. NeUmanN

F. WOLF

K. YoSHIDA

\section{SUPPORTING INSTITUTIONS}

UNIVERSITY OF BRITISH COLUMBIA CALIFORNIA INSTITUTE OF TECHNOLOGY

UNIVERSITY OF CALIFORNIA

MONTANA STATE UNIVERSITY

UNIVERSITY OF NEVADA, RENO

NEW MEXICO STATE UNIVERSITY

OREGON STATE UNIVERSITY

UNIVERSITY OF OREGON
UNIVERSITY OF SOUTHERN CALIFORNIA

STANFORD UNIVERSITY

UNIVERSITY OF HAWAII

UNIVERSITY OF TOKYO

UNIVERSITY OF UTAH

WASHINGTON STATE UNIVERSITY

UNIVERSITY OF WASHINGTON 


\section{Pacific Journal of Mathematics}

\section{Vol. 80, No. $1 \quad$ September, 1979}

Jeroen Bruijning and Jun-iti Nagata, A characterization of covering dimension by

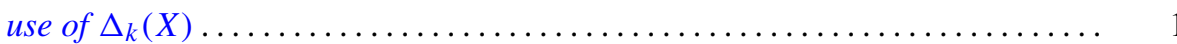

John J. Buoni and Albert Jonathan Klein, On the generalized Calkin algebra ...... 9

Thomas Ashland Chapman, Homotopy conditions which detect simple homotopy

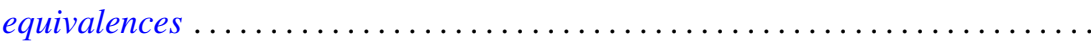

John Albert Chatfield, Solution for an integral equation with continuous interval

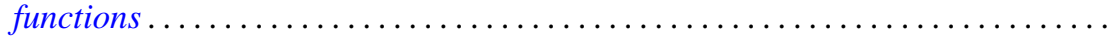

Ajit Kaur Chilana and Ajay Kumar, Spectral synthesis in Segal algebras on

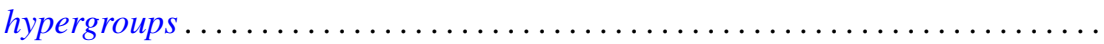

Lung O. Chung, Jiang Luh and Anthony N. Richoux, Derivations and

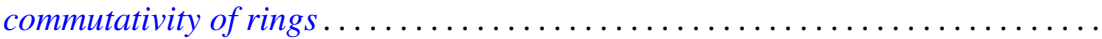

Michael George Cowling and Paul Rodway, Restrictions of certain function spaces to closed subgroups of locally compact groups .....................

David Dixon, The fundamental divisor of normal double points of surfaces........

Hans Georg Feichtinger, Colin C. Graham and Eric Howard Lakien,

Nonfactorization in commutative, weakly selfadjoint Banach algebras . . . . . . .

Michael Freedman, Cancelling 1-handles and some topological imbeddings ....... .

Frank E., III Gerth, The Iwasawa invariant $\mu$ for quadratic fields . . . . . . . . . . . . . .

Maurice Gilmore, Three-dimensional open books constructed from the identity

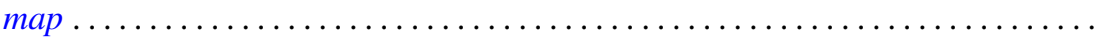

Stanley P. Gudder, A Radon-Nikodým theorem for $*$-algebras .

Peter Wamer Harley, III and George Frank McNulty, When is a point Borel? .

Charles Henry Heiberg, Fourier series with bounded convolution powers . .

Rebecca A. Herb, Characters of averaged discrete series on semisimple real Lie

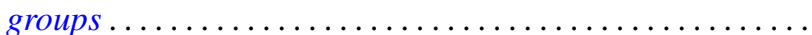

Hideo Imai, On singular indices of rotation free densities . .

Sushil Jajodia, On 2-dimensional CW-complexes with a single 2-cell . . .

Herbert Meyer Kamowitz, Compact operators of the form $u C_{\varphi}$

Matthew Liu and Billy E. Rhoades, Some properties of the Chebyshev method...

213

George Edgar Parker, Semigroups of continuous transformations and generating

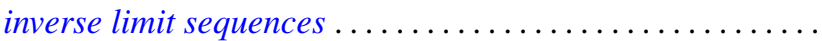

Samuel Murray Rankin, III, Oscillation results for a nonhomogeneous

equation ...

Martin Scharlemann, Transverse Whitehead triangulations ...

Gary Joseph Sherman, A lower bound for the number of conjugacy classes in a

finite nilpotent group

Richard Arthur Shoop, The Lebesgue constants for $\left(f, d_{n}\right)$-summability .

Stuart Jay Sidney, Functions which operate on the real part of a uniform

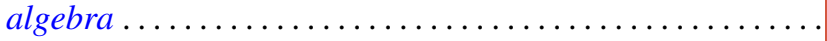

Tim Eden Traynor, The group-valued Lebesgue decomposition 